\title{
VOYAGES CHEZ LES MORTS D'EUGÈNE IONESCO : EXEMPLE D'UN DRAME À STATIONS
}

\author{
Tomasz KACZMAREK \\ Université de Łódź (Pologne)
}

\begin{abstract}
En): In this article we study the last completed piece of Eugène Ionesco Journeys Among the Dead in the perspective of expressionist aesthetics. The French playwright uses the form of a station drama in the same way as August Strindberg, precursor of the German movement, which allows him to express his anxieties and phobias. The protagonist, who is the double of the writer, sets off in the world of darkness to find his lost identity and to find his mother who he had abandoned. His wandering represents real ordeal within which he talks with the dead to get even (as with his father) or seek forgiveness, as with his mother.
\end{abstract}

Key words (En): Eugène Ionesco; August Strindberg; expressionism; station drama; onirism

Mots-clés (Fr) : Eugène Ionesco ; August Strindberg ; expressionnisme ; drame à stations ; onirisme

L'expressionnisme a été un mouvement bien limité géographiquement qui faisait florès, avant tout, dans les pays germaniques. Jean-Michel Palmier constate à ce sujet: «en France, on ne trouve pas d'équivalent de ce mouvement» (PALMIER, 1978 : 117). Il est sans aucun doute vrai que ce courant n'a pas affecté l'Hexagone comme cela a été le cas en Europe Centrale. Néanmoins, hormis les similitudes patentes en peinture (fauvisme ou cubisme), on remarque des tendances comparables dans le domaine de la mise en scène, certains n'hésitant pas à les qualifier d' «expressionnisme français » (JOMARON, 1992: 767-768). Pour ce qui est de la littérature dramatique, on ne se hasarde pas à chercher des parallèles avec l'expressionnisme, exception faite des travaux de Maurice Gravier. Celui-ci a tenté de rapprocher les œuvres de certains auteurs de l'entre-deux-guerres (Henri-René Lenormand, Jean-Victor Pellerin, Simon Gantillon) de l'esthétique allemande par l'intermédiaire du théâtre d'August Strindberg (1849-1912) qui a laissé une empreinte indélébile sur les avant-gardistes d'outre-Rhin (GRAVIER, 1962 : 45-60). De fait, si l'on voulait partir à la recherche d'éléments expressionnistes dans des textes de dramaturges français, force nous est de tenir compte de l'influence qu'a jouée sur certains auteurs la dramaturgie du Suédois, celle, en particulier, de l'aprèsInferno. On admire les «pièces mystiques » de Strindberg où la frontière entre le réel et l'imaginaire est abolie. «Les expériences oniriques [que le Scandinave] relate ont servi de laboratoire pour une des plus profondes et des plus réussies métamorphoses de la forme et du contenu qu'ait jamais connu œuvre de dramaturge » (SARRAZAC, $2018: 16$ ). On s'intéresse vivement à la forme itinérante de ses drames où se déploie l'âme du protagoniste, à la limite du réel et de l'hallucination. Le «théâtre du rêve» de celui qui sera acclamé comme un précurseur du «théâtre de l'absurde » (MAILLEFER, 2000 : 60-77) bouleversera profondément le drame moderne. Eugène O'Neil ou Arthur Adamov revendiquent ouvertement son influence sur leurs productions littéraires respectives. Parfois, 
l'empreinte de l'œuvre de Strindberg n'a pas été directe. Ionesco nie tout lien avec l'auteur du Père ${ }^{1}$ : «on me prouva que j'étais très influencé par Strindberg. Cela m'obligea à lire le dramaturge scandinave : je me rendis compte que c'était vrai » (MAILLEFER, 2000 : 60). Qui plus est, Ionesco ne pouvait pas ignorer l'œuvre du Suédois que son ami Arthur Adamov révérait à partir des années 1950. Maurice Gravier $^{2}$ a bien démontré les ressemblances existant entre Strindberg et Ionesco ${ }^{3}$, en comparant Le Songe et Le Chemin de Damas de ce premier avec La Soif et la Faim (1966). On s'est penché à juste titre sur la dimension onirique ${ }^{4}$ des œuvres de l'auteur de La Leçon, mais dans ses derniers drames, et plus particulièrement, dans L'Homme aux valises (1975) et Voyages chez les morts (1980), on constate la matrice du Stationendrama, type de drame pratiqué par les expressionnistes où l'on relate l'errance d'un personnage souffrant, «dévidant tous ses rapports subjectifs avec la réalité, c'est-à-dire [voulant] se libérer de 'l'indicible' qui stagne derrière l'apparence du réel » (RICHARD, 2012 : 124). De fait, à voir de plus près, Ionesco semble renouer avec la tradition expressionniste ou, tout au moins, puiser dans l'esthétique thêâtrale d'August Strindberg, celle qui a posé les jalons de l'expression subjective de la génération allemande. Cette hypothèse heuristique ne vise point à prouver que Ionesco s'inspire directement de la poétique expressionniste. Qui plus est, on est loin de faire du dramaturge un porte-drapeau de l'esthétique que la France a longtemps ignorée. Comme l'expressionnisme naît de la crise ontologique au tournant du $\mathrm{XX}^{\mathrm{e}}$ siècle, il est curieux de noter des réactions analogues en France dans les décennies qui succèdent à la Deuxième Guerre mondiale. Il serait intéressant à ce propos d'étudier les résurgences expressionnistes qui se manifestent avant tout dans Voyages chez les morts ${ }^{5}$ de Ionesco dont la forme de drame itinérant lui permet d'exprimer les dessous de son âme inquiète.

Avant d'aborder la problématique de l'article, il serait légitime de retracer ne serait-ce que sommairement les caractéristiques essentielles du drame expressionniste. L'expressionnisme surgit comme un mouvement artistique, à partir de la peinture, qui va toucher tous les arts sans pour autant créer un programme

\footnotetext{
${ }^{1}$ Dans ses Notes et contre-notes, Ionesco considère Strindberg comme un auteur «insuffisant, maladroit » (IONESCO, $1966: 52,55)$.

${ }^{2}$ Cf. Gravier Maurice (1975), Strindberg et Ionesco, in : SMEDMARK Carl Reinhold, (éd.), Strindberg and Modern Theatre, the Strindberg Society, Stockholm.

${ }^{3}$ Pour en savoir plus, se rapporter à : GuÉRIN Jeanyves, (2012), (éd.), Dictionnaire Eugène Ionesco, Paris, Honoré Champion, coll. « Dictionnaires et références ».

${ }^{4}$ La problématique a bien été étudiée dans plusieurs ouvrages ou articles. Voir à ce propos par exemple : FÉAL Gisèle (2001), Ionesco - un théâtre onirique, Éditions Imago ; Chavanne Philippe (2015), La Dramaturgie onirique d'Eugène Ionesco, Edilivres ; MACÉ-BERBIER Nathalie (1997), "Onirisme et dramaturgie chez Ionesco (L'Homme aux valises et Voyages chez les morts) ", Revue d'Histoire littéraire de la France, 97 Année, № 4, pp. 649-667.

${ }^{5}$ Nous avons jugé opportun de limiter notre étude aux Voyages chez les morts, tout en omettant à bon escient L'Homme aux valises, car ce premier drame constitue un vrai testament de Ionesco qui le rapproche plus explicitement de Strindberg. Au demeurant, l'auteur même attire notre attention sur les différences entre ces deux pièces, ce qui a directement influé sur notre décision : "Dans L'Homme aux valises, le langage essaie de s'adapter aux images oniriques, le langage, c'est-à-dire la parole, le dialogue. Ce n'est pas parfait parce que j'ai triché parfois, inconsciemment ou consciemment. Dans la pièce que j'écris maintenant [Voyages chez les morts], j'essaie de ne pas toucher à ce que j'ai vu, à ce que j'ai entendu, à ce que j'ai dit dans le rêve » (IONESCO, $2016: 28$ ).
} 
unique et cohérent. En dépit de multiples différences au sein même des groupes artistiques, on retrouve certains thèmes et recherches formelles qui leur sont communs: «abstraction, violence des couleurs et des éclairages, négation de la réalité, développements oniriques et apocalyptiques, rupture avec les formes classiques, souci d'exprimer l'intériorité et son déchirement » (PALMIER, 1978: 118). Loin d'être homogène, le drame expressionniste, tout comme la peinture, consiste en la projection d'une subjectivité qui vise à déformer la réalité pour provoquer une émotion forte chez le spectateur. Pour atteindre à l'expressivité la plus intense, les artistes recourent à l'outrance, tout en favorisant l'emphase et l'hyperbole. L'esthétique du «cri » et de l'excès provient d'une vision pessimiste et apocalyptique qu'ils ont de leur époque. Dans ce contexte, l'expressionnisme n'est pas seulement un phénomène esthétique, mais aussi (ou avant tout) une réponse virulente à une humanité matérialiste. Dès lors, faisant abstraction de la réalité physique, les auteurs privilégient un subjectivisme parfois démesuré où les états d'âme du dramaturge peuvent s'étaler en toute liberté. Dans cet "espace intérieur », où règne sans partage le «moi » du protagoniste (qui est le double de l'écrivain), tout est criblé par son esprit dérangé, tout est subordonné aux fluctuations de son âme égarée. On ne connaît son monde que par le biais de sa perception frelatée qui se dérobe aux contraintes extérieures de l'art dit réaliste. De fait, les auteurs renoncent à la psychologie traditionnelle. À l'instar de Strindberg, on rejette la notion de «caractère» pour favoriser «la psychologie des profondeurs » des personnages. Cette approche trahit « la crise du sujet» qui, loin de pouvoir être cerné comme une entité indivisible, échappe à toute analyse réductrice. La fonction du personnage change aussi. Extrait de son entourage social, il perd son identité au point de perdre son existence propre, «il n'est que l'attribut du héros principal, l'incarnation symbolique des possibilités de celui-ci, l'expression de son inconscient, lequel inconscient n'était pas saisissable dans le réel » (STEFFENS, 1993: 159). Les comparses apparaissent comme des manifestations psychiques du protagoniste, ils tournent autour de lui, tels des satellites, tout en symbolisant les forces contradictoires de son âme. Dans ce contexte, il n'est pas étonnant de voir dans le drame expressionniste l'atomisation du personnage qui, comme le voulait l'auteur de Mademoiselle Julie, se dédouble ou se multiplie à volonté. C'est dire qu'au centre du drame se trouve «le moi confessant » du poète qui chante sa détresse, ce même moi étant amené à rencontrer ses propres fantômes. Il est incontestable que ce type de drame introduit un nouveau paradigme qui déborde les formes canoniques, son subjectivisme effréné entrainnant inévitablement de nouvelles solutions structurelles. Or, la dimension visionnaire et onirique exige la refonte dramatique qui remet en cause l'action même. Celle-ci disparaît au profit de l'enregistrement arbitraire d'images floues, sorties de l'esprit de l'homme en proie à la souffrance. S'inspirant sans aucun doute des « Passions » du Moyen Âge, les expressionnistes privilégient la structure fractale, celle des stations qui rend compte par étapes de la vie du protagoniste et de son évolution mentale (c'est ainsi qu'ils sécularisent le matériau religieux) : « le récit biblique de la Passion est centré sur la personne de Jésus, le drame expressionniste s'attache à décrire le monde extérieur comme une concentration de forces hostiles, mais ce monde extérieur n'existe que par son rapport au personnage central, dont il est 
comme une projection » (MAZELLIER-GRÜNBECK, 1994 : 66). C'est pourquoi le monde intérieur du poète est déformé au travers de ses hallucinations et de son délire :

Tandis que l'impressionnisme cherche à réaliser l'objet artistique à partir du sujet individuel, différent («vulgaire », pour ainsi dire), la tâche de l'expressionnisme n'est plus dans la réalisation de l'idée. Contrairement à celle de l'impressionnisme, la tâche de l'expressionnisme n'est donc plus liée à un objet mais uniquement au sujet de l'artiste qui, à la fin de réalisation, utilise tous les objets quels qu'ils soient, les associe et les amalgame, en déforme et déporte la signification réelle. D'où, en peinture, les distorsions des formes ou leurs désagrégations, arbitraires apparemment mais en vérité absolument nécessaires; d'où les substitutions et les translations des concepts et images en poésie, les caractères «faussement vrais» des personnages dramatiques ou romanesques (RHEINER, $1995: 104)$.

Ce subjectivisme n'est pas étranger à Ionesco, son œuvre s'inspirant de ses propres expériences et de ses rêves qu'il transcrit le plus fidèlement possible, par exemple, dans Journal en miettes. Quand on relit l'avertissement que Strindberg formule au sujet du Songe, un jeu de rêves (1901), on retrouve la proximité d'esprit entre Ionesco et l'auteur scandinave. Ce dernier précise qu'il a cherché dans son œuvre :

à imiter la forme incohérente mais apparemment logique du rêve. Tout peut arriver, tout est possible et vraisemblable. Temps et espace n'existent plus. À partir d'une base réelle insignifiante, l'auteur donne libre cours à son imagination qui multiplie les lieux et les actions en un mélange de souvenirs, d'expériences vécues, de libre fantaisie, d'absurdités et d'improvisations. Les personnages se dédoublent et se multiplient, s'évanouissent et se condensent, se dissolvent et se reconstituent. Mais une conscience suprême les domine tous : celle du rêve (STRINDBERG, 1964 : 137).

Ionesco aurait pu souscrire à ce credo artistique, car lui-même n'hésite pas à déclarer que «tout est permis au théâtre ». Il désire pour son œuvre « une scène intime » apte à « incarner les personnages, mais aussi à matérialiser des angoisses, des présences intérieures. Il est donc non seulement permis, mais recommandé de faire jouer les accessoires, faire vivre les objets, animer les décors, concrétiser les symboles » (IONESCO, 1966 : 63). Le dramaturge n'arrête pas de lancer l'idée de la création d'un autre type de théâtre :

un théâtre pas symboliste, mais symbolique ; non pas allégorique, mais mythique ; ayant sa source dans nos angoisses éternelles; un théâtre où l'invisible devient visible, où l'idée se fait image concrète, réalité, où le problème prend chair ; où l'angoisse est là, évidente, énorme (IONESCO, 1966 : 311-312)

L'écrivain s'efforce de réaliser ces postulats dans sa dernière pièce achevée. Dans le programme édité à l'occasion de la mise en scène de Voyages chez les morts à l'Odéon en 1984, Ionesco ne manque pas de préciser : «Quand je veux raconter 
ma vie... c'est une errance que je raconte » (IONESCO, 1991: 1858) et dans une interview accordée à Hervé Gauville, il s'empresse de répéter que son dernier drame n'est que l'histoire de sa vie «mais à l'envers », vue à travers le rêve : "comme un négatif photographique. Il y a aussi un voyage qui est à la fois retour aux sources et initiation, mais qui n'aboutit nulle part. Une errance. L'existence est une errance dans l'absurde » (IONESCO, 1991 : 1863). Le dramaturge souligne à maintes reprises le caractère autobiographique du drame qu'il agrémente librement de ses rêves incarnant ses propres angoisses et phobies. Jean, «existant spécial » qui est incontestablement le double de l'auteur et le héros central du drame, se met en marche, tels les personnages strindbergiens, à la recherche de sa Mère et, « à travers elle, trouver la Mère ${ }^{6}$, mythique $e^{7}$ liée à la vie et à la mort » (IONESCO, 1991 : 1864). Ionesco choisit la forme du drame itinérant, car «l'échelonnement des stations permet de concentrer sur quelques moments cruciaux le déroulement épique d'une vie » (SARRAZAC, 2012: 136). En cours de route, le protagoniste rencontre plusieurs personnages du clan maternel et du clan paternel, deux groupes en lice qui se vouent une haine sincère et réciproque. Certains d'entre eux sont déjà morts, d'autres se trouvent suspendus dans un vide, sortis ne serait-ce que momentanément de la mémoire du personnage principal. Cette quête dans le royaume des ombres fait revivre à Jean un passé souvent douloureux, lui permet de prendre sa revanche sur ses ennemis jurés, mais elle réveille aussi le sentiment de la culpabilité surtout visà-vis de sa Mère et de son meilleur ami Alexandre (le prototype d'Arthur Adamov). Le protagoniste se questionne sur les raisons pour lesquelles il entreprend ce long voyage à travers des temps révolus, tout en s'entretenant avec des personnes disparues depuis longtemps, et devine, non sans horreur, que la visite des défunts annonce selon toute probabilité l'approche imminente de sa propre mort. Il le confirme dans le deuxième dialogue avec son père :

Depuis des années tu es toujours dans mes rêves, et toi, et ta femme et ma mère et tes beauxfrères. Je n'avais plus rêvé de vous tous pendant des années, des dizaines d'années. Que signifie ce retour vers vous? Est-ce que je vais bientôt vous rejoindre ? On n'a pas fini de régler nos comptes? Toujours en revenir à ces débuts effroyables (IONESCO, $2016: 61$ ).

C'est aussi l'inquiétude de Ionesco qui, arrivé à l'âge de soixante-et-onze ans, éprouve le désir de faire le bilan de son existence, comme s'il voulait se préparer à son ultime périple. C'est peut-être dans cette œuvre que l'écrivain révèle courageusement les faits parfois les plus intimes de sa vie privée. Il dote son héros du nom de $\mathrm{Jean}^{8}$ qui, comme le souligne Marie-Claude Hubert, renvoie au roumain Ion $($ Ion + escu $=$ Ionescu $)$ et qui est l'équivalent du prénom français. Cette projection autobiographique évidente, contrairement à ce qui se passe dans L'Homme aux valises où les questions politiques et sociales côtoient celles de l'égarement mental du personnage principal, se focalise uniquement sur le drame

\footnotetext{
${ }^{6}$ Nous utilisons des majuscules quand nous nous rapportons aux personnages (Père, Mère) de la pièce et des minuscules dans le cas des parents de l'écrivain (père, mère).

${ }^{7}$ Souligné dans le texte.

${ }^{8}$ C'est le même prénom que Ionesco donne au protagoniste de La Soif et la Faim.
} 
intérieur de l'écrivain. Ici, Ionesco renonce au «je » qui domine dans ses textes hautement confessionnels comme Journal en miettes ou Présent passé, passé présent, pour investir son héros de ses propres traits et pour scruter les méandres de son propre psychisme incertain par le biais de son «sosie ». La forme dramatique lui permet de mieux exprimer ses doutes et ses angoisses, ses rancunes et ses remords, ainsi que de rendre compte, comme l'a fait Strindberg bien avant lui, de la conflictualité de l'existence humaine qui s'avère un enfer insupportable. Il n'oublie pas pour autant les forces contradictoires qui siègent dans l'âme de l'homme « jetédans-ce-monde » et sans secours. Comme l'auteur du Père, le dramaturge français semble entamer une vivisection torturante de son être qui n'est, pour reprendre le titre d'une œuvre de François Mauriac, qu' « un nœud de vipères ». C'est pourquoi Ionesco recourt au dialogue dont le caractère agonistique témoigne d'une lutte aussi constante qu' absurde.

En lisant ce drame, on a l'impression que les étapes (elles sont au total 18) que Jean traverse se succèdent sans aucune liaison. Ionesco ne numérote même pas les haltes qui signent le parcours initiatique du protagoniste. Seuls les astérisques séparent une «station»d'une autre, comme si l'ordre de leur déroulement était laissé au hasard. Pourtant, la construction dramatique, si éparpillée qu'elle puisse paraître, observe une progression interne qui relate une quête spirituelle du héros central. C'est elle qui constitue le pivot de l'agencement des faits qui se suivent. «L'irradiation du moi » de l'auteur détermine de cette manière l'enchaînement des scènes qui, comme dans le rêve, s'étalent sans lien logique et raisonnable. Tout se passe conformément à l'évolution de l'âme du protagoniste qui rêve. C'est à lui, image de l'écrivain, qu'incombe le rôle de celui qui tient la baguette de chef d'orchestre. Dans cette perspective, la pièce semble renouer avec le Ich-drama expressionniste, où «l'action qui se déroule sur la scène n'est que le reflet d'un drame plus profond [se jouant] dans la conscience du personnage » (PALMIER, 1980 : 20). Malgré l'absence de lien causal entre les stations, la présence de Jean garantit l'homogénéité de l'œuvre, minée par des forces centrifuges, car, conformément aux prescriptions des dramaturges expressionnistes, « dans le 'drame à stations', le personnage principal est le seul qui assure une certaine unité et continuité de l'action dramatique »(GOUDÉ, 1999 : 322).

Ainsi, dans les six premières scènes, Jean est confronté à deux coteries : maternelle et paternelle. À partir de la scène VII jusqu'à la scène XII, il se penche sur des questions ontologiques : il cherche le sens de sa vie et de son œuvre, tout en pressentant l'approche de sa mort. Les scènes XIII à XVI font revivre l'enfance joyeuse du personnage, tandis que la scène XVII constitue le Jugement dernier qui finit par un non-lieu. La dernière scène, qui clôt définitivement les pérégrinations de Jean, n'est qu'une ultime expression de sa pensée ombrageuse. Marie-Claude Hubert remarque à juste titre que lesdites scènes sont construites en écho, les unes renvoyant aux autres. Le drame porte en effet le sous-titre Thèmes et variations, ce qui souligne, aux yeux de Jean-Pierre Sarrazac, sa forme rhapsodique, apte à relater le drame de la vie (non seulement du protagoniste, mais de chaque homme) dans toute son incohérence. Il s'agit bien des thèmes qui taraudent l'esprit de Jean. On pourrait évoquer à ce propos l' animosité que Ionesco exprimait à l'égard de son père autoritaire et coléreux, la figure de ce dernier étant déformée par ses travers et le 
ressentiment de son fils. Dans les scènes II, IV, VI et XII nous assistons à différentes tentatives de règlements de comptes du héros central avec son père. Le premier ne tuera pas le second dans un accès de colère, comme dans le drame expressionniste, mais il lui déclarera sa haine, la même que la génération allemande des «jeunes » vouait à la caste des « vieillards » usurpateurs et oppresseurs. Jean revoit son Père dans ses souvenirs, tantôt vivant, tantôt mort, comme si Ionesco désirait enfin régler un différend avec son propre père. Il lui reproche son opportunisme politique et surtout sa violence :

je suis toujours l'enfant malheureux que tu opprimais, que tu battais. Tu m'injuriais à cause de ma mère qui ne t'avait fait aucun mal et que tu as abandonnée. Heureusement que j'ai pu fuir de chez toi à dix-sept ans. Que m' aurait apporté un père comme toi, qui frappait ses domestiques ? (IONESCO, $2016: 62$ ).

La liste des reproches ne s'arrête pas là. Le fils ne peut pas pardonner à son procréateur le divorce et le remariage avec $\mathrm{M}^{\mathrm{me}}$ Simpson. Il semble intransigeant mais dans la scène VI, le protagoniste se dédouble, comme s'il voulait prendre du recul, et c'est à Jean II de réprimander le vieillard cynique tandis que Jean I écoute la conversation immobile. C'est au cours de ce dialogue, qui ressemble à s'y méprendre à la manière de Strindberg, que nous apprenons la défaite morale du père :

LE PÈRE : Malgré moi, tu as fait ce que tu as voulu dans la vie. Je rêvais pour toi un autre destin, une autre carrière : grand fonctionnaire politique, ou bien général, ou bien ingénieur chimiste. Tu n'as pas voulu m'obéir. Je sais, je ne lui en veux plus, c'est ta mère qui poussait dans d'autres directions.

JEAN : Tu lui en veux encore ! Tu lui en voudras pour l'éternité. Tant que tu lui en voudras, tu n'iras pas au paradis. Je suis venu là, je reste dans mon fauteuil pour répondre à tes questions.

LE PÈRE : Ne m'accable pas ! Je dois reconnaître que tu as fait une belle carrière, tu as réussi dans la vie [...] (IONESCO, $2016: 85-86)$.

Cependant la victoire de Jean est de courte durée. Le vieillard lui demande de montrer ses œuvres et, comme dans un des rêves racontés par Ionesco dans $U n$ homme en question, Jean sort d'un tiroir du papier jauni, des cahiers en morceaux, des bouts de fil de fer rouillés, des petits chiffons sales. C'est tout ce qui reste de sa gloire passée. Alors, le protagoniste se rend compte que tout est vanité, même sa révolte contre la tyrannie paternelle s'avère inutile dans le royaume des ténèbres.

Le protagoniste se perd dans un espace chaotique. Tel L'Inconnu strindbergien, il peine à reconnaître les personnages qu'il côtoie. Ceux-ci prennent les traits de spectres. Ce monde du rêve demeure à tout moment plastique, chaque image se transformant en une image différente. En témoigne la scène VIII où, comme dans Le Songe de Strindberg, la rue Claude-Terrasse (adresse où l'auteur vivait avec sa femme), devient à l'improviste le moulin de La Chapelle-Anthenaise (Ionesco y a passé une partie de son enfance) pour, à la fin, laisser la place au château de Cerisyla-Salle (l'endroit des colloques dédiés à l'œuvre du dramaturge). Ainsi, les images 
soumises à de brusques variations se conforment aux sentiments fluctuants du rêveur. Néanmoins, dans ce labyrinthe onirique au niveau de l'espace, du temps et du langage, le fil conducteur de la pièce est toujours là. C'est la recherche de la Mère que le protagoniste, tout comme Ionesco, a laissée mourir seule, ce motif principal assurant « la cohérence de l'incohérence » (IONESCO, $1991: 1862$ ).

Comme dans Le Chemin de Damas, L'Inconnu rencontre des créatures étranges qui reflètent ses tourments moraux, Jean s'entretient avec des femmes en qui il a du mal à discerner les traits de sa Mère, mais qui, en quelque sorte, «matérialisent » à plusieurs reprises les remords du fils qui a abandonné sa génitrice. Jean recherche avec la plus grande affliction sa Mère qu'il n'avait pas vue depuis longtemps. Il se rend à différents endroits, mais à chaque fois il arrive trop tard. Dans la scène III, le protagoniste pénètre en rampant à plat ventre dans un antre obscur, une sorte de tombeau sans fenêtres, où habitent deux femmes qui portent des masques ressemblant à sa Mère. Quand Jean les aborde, il jalonne les étapes de sa propre anxiété :

LA DEUXIÈME FEMME : Elle est peut-être partie pour un certain temps.

JEAN : (anxieux et attristé) Peut-être est-elle partie justement parce qu'elle sentait que j'allais venir? Je ne lui ai jamais fait de tort, sauf par omission.

LA PREMIÈRE FEMME : Ce sont là des problèmes où nous ne pouvons pas intervenir.

LA DEUXIÈME FEMME : Elle est peut-être partie dans l'autre province voir une amie, Julienne. Elle a un beau château, tout noir. Elle avait passé des moments heureux dans ce château, elle voulait le revoir avant sa démolition.

JEAN : Le château noir ? Vous voulez dire le château blanc ?

LA PREMIÈRE FEMME : Elle était pourtant bien là, il y a un instant. Elle est peut-être partie pour toujours (IONESCO, $2016: 59-60$ ).

Jean reprend aussitôt son chemin. Sur sa route, il rencontre sa marâtre et ses frères, son ancien ami, sa sœur et sa propre femme ainsi que d'autres anonymes, témoins de son calvaire, et fait face à d'autres femmes encore, qui lui rappellent les torts causés par lui à sa Mère. Bourrelé de remords, il cherche des excuses (scène XI) devant une vieille qu'il prend pour sa Mère, mais qui se présente comme sa grand-mère maternelle : " $\mathrm{J}$ 'ai des circonstances atténuantes, plusieurs fois, $\mathrm{j}$ 'ai essayé de venir. J'étais dans la rue, pour venir vous voir. En fait, la rue n'était qu'une impasse, je devais rebrousser chemin. Je faisais des détours, je traversais d'autres rues, qui étaient toujours des impasses » (IONESCO, 2016 : 127). Le protagoniste tente une dernière fois de s'expliquer dans la pénultième scène (XVII), devant sa Mère enfin retrouvée, mais ses dérobades ne convainquent point la vieille qui lui montre ses griffes :

JEAN : Je t'ai cherchée partout.

LA VIEILLE : Tu n'as pas voulu vraiment, tu étais dans tes palais, dans tes châteaux avec tes belles, tu n'as pas pensé à moi, tu habitais la maison de ton père, qui était beaucoup plus riche.

JEAN : Il est mort lui aussi depuis longtemps.

LA VIEILLE : Mais grâce à ses richesses, il a payé aux églises et il a une maison convenable pour les morts, il a des meubles et de la nourriture, la vie n'est pas juste, la mort n'est pas juste non plus ; et toi ? oui, oui, oui, tu faisais semblant de me chercher. 
JEAN : Je t'ai cherchée dans tous les cimetières, dans les maisons de vieillards, chez ta sœur et ta cousine, chez les vivants et chez les morts, je t'ai cherchée dans les registres des églises et je n'ai pas trouvé ton nom, maman.

LA VIEILLE : C'est parce que tu n'as jamais fait dire des messes pour moi quand tu me cherchais dans cette maison, tu ne regardais jamais en haut, mais seulement le plancher pourri et tu t'enfuyais vite, tu avais peur, tu avais honte [...] Où suis-je maintenant ? dans la fosse commune, mais j'ai pris garde et je me suis cachée au-dessus du plafond et c'est pour cela que cette maison n'est pas tombée en ruine malgré sa vétusté. Et je ferai trembler les fondations et j'y mettrai le désordre (IONESCO, $2016: 185-186$ ).

Quand elle menace de bouleverser l'ordre, elle pense à se venger impitoyablement sur ses adversaires, tout en désirant ébranler le psychisme du fils. Ionesco donne ainsi à sa mère une voix dont elle ne disposait pas de son vivant. Il l'imagine comme une justicière implacable qui n'épargne pas non plus les mots durs à son fils, malgré les promesses de celui-ci de lui ériger une belle sépulture. Commence alors une scène rappelant le Jugement dernier. La Mère se montre particulièrement féroce. Elle prononce accusations et condamnations, elle exécute aussi ses verdicts. Le Capitaine, frère de la deuxième femme du Père, sera jugé le premier. La Vieille lui serre la gorge, en lui plongeant l'autre main dans le crâne. Elle lui prend son sabre qu'elle lui enfonce dans le ventre puis elle lui arrache l'œil droit. $\mathrm{M}^{\mathrm{me}}$ Simpson (prototype de la marâtre de Ionesco) partagera le sort de son prédécesseur : la Mère lui enlève son faux-nez et ses fards derrière lesquels se cache une vieille bossue. Elle lui donne un coup de pied et gifle fortement son deuxième frère, le Haut Fonctionnaire. Quant au Père, la Vieille ordonne à la Tzigane, la dernière amante en date de l'inculpé, de le trainer par terre et de le pendre. Une fois sa vengeance accomplie, la Mère prend les traits d'une belle jeune femme qui se cachait derrière ses hardes et son faux nez. Pourtant, dans la mort, tout est aboli, ni la haine, ni l'amour n'existent. C'est alors que tous se relèvent et quittent le plateau en riant.

Cette descente dans le mental infernal devrait donner au protagoniste l'occasion de percer le mystère caché perfidement aux humains, mais elle lui révèle la vanité de toute chose et, qui plus est, l'indifférence absolue de l'univers face à son désespoir. Jean-Ionesco cherche éperdument à résoudre l'énigme de la vie et de la mort, tout en sachant que la langue (logos) s'avère en l'occurrence défaillante. Ceci ne l'empêche pas de continuer sa quête enfiévrée, comme si c'était la dernière chose à faire dans son existence précaire. C'est pour cette raison qu'il se penche avec obstination sur son passé et le revit dans une dimension onirique, tout en désirant le déchiffrer comme un expert en matière de perturbations de l'âme égarée. On se rappelle à ce propos le dialogue que l'Inconnu du Chemin de Damas mène avec la Dame :

LA DAME : Pourquoi as-tu crié pendant le sommeil?

L'INCONNU : J'avais un rêve...

LA DAME : Un véritable rêve?

L'INCONNU : D'une terrifiante réalité... mais vois-tu la malédiction : J'éprouve le besoin de le raconter et à qui le raconterais-je, sinon à toi ? Mais je ne puis le raconter, sans me heurter à la porte de la chambre défendue... 
LA DAME : Le passé...

L'INCONNU : Oui !

LA DAME : C'est déjà une folie d'avoir comme cela une chambre secrète.

L'INCONNU : Sûrement (STRINDBERG, $1983: 250$ ).

Il s'ensuit que le rêve serait capable de donner accès à la chambre secrète de tout individu, car par le rêve notre âme peut enfin connaître ses propres mystères cachés dans notre inconscient. La méthode de Strindberg anticipe celle de Freud, mais on n'y découvre point les vertus curatives de celle-ci. « Pessimiste et sceptique comme les disciples de Bouddha, Strindberg s'abîme dans les profondeurs du rêve, avec l'espoir de dépasser ainsi le monde des apparences » (GRAVIER, 1949 : 153). Ionesco désire, lui aussi, pénétrer l'écorce de la matérialité en construisant un monde fragile aux formes inconsistantes et fugaces, mais il sait bien que tout se dérobe à une quelconque compréhension. La seule vérité dont l'homme puisse être sûr, c'est sa mort. Et Jean-Ionesco sent que sa propre mort est proche. En écho au dialogue entre la Dame et l'Inconnu, le dramaturge français imagine dans la scène VIII un bref entretien entre le protagoniste et la Femme au cours duquel Jean semble ne plus se faire d'illusions sur le motif de son long voyage :

JEAN : [...] C'est le plus grand voyage. Mais vous me dites que ce monde n'est qu'approximativement vrai, qu'à peu près vrai, alors, où est le vrai, le tout à fait vrai ?

LA FEMME : L'air pur du tout à fait vrai, vous le sentez déjà ici. Cependant ce n'est que l'antichambre du vrai qui ne bouge pas. Je dois vous emmener plus loin. Ne vous effrayez pas, ce n'est pas mesurable, ce n'est ni long ni court, mais je dois vous emmener avec d'autres gens.

JEAN : Je m'en doutais bien, je sais qui je dois rencontrer, n'est-ce pas ?

LA FEMME : Oui vous savez (IONESCO, 2016 : 107-108).

Au terme de son itinéraire, le protagoniste, qui apparaît assis dans un fauteuil, et qui est désigné par le dramaturge dans les didascalies comme «le Récitant (ou) Jean », essaie une dernière fois (scène XVIII) de revenir sur son passé et sur son parcours initiatique. Néanmoins, quand il s'apprête à prendre la parole, il se rend compte qu'il n'est pas à même de s'exprimer clairement. Ce n'est pas que les mots lui manquent. Ceux-ci s'envolent librement comme dans l'exposé verbeux et creux d'un halluciné. Dès les premières phrases, son discours devient chaotique et confus, marqué par des pauses, moments de suspension quand le personnage s'efforce de brider le flux de sa pensée. Le protagoniste parle, évoquant pêle-mêle les endroits traversés, cherchant les mots justes pour décrire sa détresse, mais au lieu d'un monologue cohérent à travers lequel il pourrait faire part de ses tribulations, il sombre dans une allocution où les mots, proférés dans le désordre, sont dépourvus de toute substance sémantique. Il s'aperçoit que «les mots disent des choses. Les choses disent-elles des mots? » (IONESCO, $2016: 204)$. Ce long soliloque, digne des meilleurs expressionnistes, résume en quelque sorte non seulement la condition absurde du héros mais aussi celle de toute l'humanité. Jean découvre que sa recherche du mystère de la vie devait se solder par l'échec, car c'était son destin inexorable depuis sa naissance, tout comme celui de chaque être humain. Il n'est point furieux, tels les doyens du Songe de Strindberg, qui constatent avec 
ahurissement que derrière la porte, perfidement fermée à leurs yeux, ne se trouve point la clef de la « vérité ontologique », mais se cache tout simplement le « vide »: «Le vide ! C'est la réponse de l'énigme du monde ! (STRINDBERG, 2006 : 120). Tel est aussi l'enseignement que le héros tire de son errance qui n'est que, en condensé et à rebours, l'histoire de sa vie. Jean se montre résigné, ou mieux, blasé devant l'inévitable. Il pourrait encore se révolter, mais face au néant tout geste s'avèrerait dérisoire. À un moment donné, il s'adresse à son public pour dénoncer les chimères censées apaiser l'insupportable douleur existentielle : « Mesdames et messieurs qui n'existez pas, et toi public, qui es un trou noir, mon exposé contient plusieurs arguments d'importance d'où il suit que le sauveur sauvé sauvera. Tout cela c'est du foin » (IONESCO, 2016 : 203). Et il termine sa péroraison, une sorte de testament aussi ironique que pathétique, par un constat désabusé : «Je ne sais pas. Je sais seulement que j'ai gardé sur moi les bribes et les miettes des cellules. Je ne sais pas » (IONESCO, 2016 : 204). C'est ainsi que l'errance de Jean dans l'absurde touche à sa fin.

Avec le drame à stations on parvient, aux dires de Klaus Kändler, « à une pièce qui est à l'antipode du drame classique à conflit. Il décrit des situations et renonce aux imbroglios dramatiques résultant des caractères individuels » (KÄNDLER, 1995 : 124). La pièce de Ionesco, privée d'action au sens traditionnel du mot, s'articule conformément à l' enchaînement des images et des visions du protagoniste rêvant, tout en rejetant les lois de la causalité. Dans Entre la vie et le rêve l'auteur note à ce propos que «dans les rêves il n'y a pas de progression rigoureuse. On passe d'une image à l'autre, les associations se font librement » (IONESCO, 2016 : 14). C'est pourquoi les scènes semblent se suivre sans lien logique. Pourtant, la conscience du héros central domine sur le déroulement des épisodes. Dans cette perspective, Voyages chez les morts renouent avec le «théâtre du rêve » de Strindberg, où on retrouve l'espace segmenté d'un chemin de vie, le tout étant subordonné au flottement de l'âme du protagoniste. Dépourvu de son « caractère », nous assistons à l'évolution souvent incohérente de l'esprit du personnage. De fait, comme dans Le Chemin de Damas, le dramaturge français abandonne le conflit interhumain pour privilégier «la confrontation d'un homme avec ses propres fantômes, qu'il s'agisse de doubles, de projections de lui-même ou de relations intimes » (SARRAZAC, $2018: 59)$.

Ionesco tâche ainsi de projeter sur scène un drame intérieur, son propre drame dans un monde déchiqueté et désarticulé à l'image de son âme troublée. Jean, qui est le double de l'écrivain, se met en route à la recherche de sa Mère et du sens de sa vie. Chaque étape, rappelant un vrai calvaire, le marque douloureusement puisque, en avançant, il se rend de plus en plus compte de l'inutilité de ses pérégrinations. Mais c'est aussi une quête sans fin de soi-même, de son identité fragmentée à travers la multiplicité de ses propres hypostases. Comme dans le drame expressionniste, le personnage souffrant rencontre d'autres comparses qui, loin d'être des individus à part, reflètent ses angoisses et ses remords. Ce chemin d'errance est donc un chemin de repentance au cours duquel il tente de se réconcilier avec sa génitrice. C'est aussi une tentative de règlement de comptes avec le Père et 
sa «nouvelle» famille. Les stations où s'arrête Jean-Ionesco en gravissant son chemin de croix lui révèlent enfin l'approche de sa mort dont il a peur depuis l'âge de quatre ans. C'est une terrible pénitence de l'homme, ce que le dramaturge confirme en observant : «j'écris [...] pour crier ma peur de mourir, mon humiliation de mourir » (IONESCO, $1966: 309)$.

En somme, la pièce testamentaire de Ionesco, dans laquelle il se penche sur son passé, réalise pleinement ses désirs d'un nouvel art dramatique, tout en essayant d'exorciser ses craintes. Son «voyage onirique» semble se rapprocher du « laboratoire infernal » de l'auteur du Chemin de Damas. De fait, en lisant les notes de l'écrivain français sur le théâtre, on y constate maintes analogies avec les théories pré-expressionnistes de Strindberg :

Pour moi, le théâtre - le mien - est, le plus souvent, une confession ; je ne fais que des aveux [...] Pas d'intrigue, alors, pas d'architecture, pas d'énigmes à résoudre mais de l'inconnu insoluble, pas de caractères, des personnages sans identité [...] simplement une suite sans suite, un enchainement fortuit, sans relation de cause à effet, d'aventures inexplicables ou d'états émotifs, ou un enchevêtrement indescriptible, mais vivant, d'intentions, de mouvements [...] (IONESCO, $1966: 226)$.

\section{BIBLIOGRAPHIE}

FAUCHEREAU Serge (2001), Expressionnisme, dada, surréalisme et autres ismes, Paris, Denoël.

GodÉ Maurice (1999), L'expressionnisme, Paris, Presses Universitaires de France.

GRAVIER Maurice (1949), Strindberg et le théâtre moderne. I. L'Allemagne, Lyon, Bibliothèque de la Société des études germaniques.

GRAVIER Maurice (1962), Strindberg and French Drama/Strindberg et les Dramaturges français, World Theatre 11-Le théâtre dans le monde, $\mathrm{n}^{\mathrm{o}} 1$, Bruxelles, p. 45-60.

IONESCO Eugène (1966), Notes et contre-notes, Paris, Gallimard.

IONESCO Eugène (1991), Théâtre complet, édition présentée, établie et annotée par Emmanuel Jacquart, Bibliothèque de la Pléiade, Paris, Gallimard.

IONESCO Eugène (2016), Voyages chez les morts, édition présentée, établie et annotée par Marie-Claude Hubert, Paris, Gallimard.

JOMARON Jacqueline de (1992), (éd.) Le théâtre en France du Moyen Âge à nos jours, Paris, Armand Collin, coll. «La Pochothèque ».

KäNDLER Klaus (1995), «Une structure fondamentale : le drame à stations », trad. André Combes, Lionel Richard, Obliques 6-7, p. 123-128.

MAILLEFER Jean-Marie (2000), Un précurseur du théâtre de l'absurde, Europe, $\mathrm{n}^{\circ}$ 858, Paris, 60-77.

MAZELLIER-GRÜNBECK Catherine (1994), Le théâtre expressionniste et le sacré. Paris, Peter Lang.

PALMIER Jean-Michel (1978), L'expressionnisme comme révolte, Paris, Payot.

PALMIER Jean-Michel (1980), L'expressionnisme et les arts (2. Peinture-théâtrecinéma), Paris, Payot.

RHEINER Walther (1995), «Expressionnisme et le théâtre », trad. Victor Morel, Lionel Richard. Obliques 6-7, p. 101-104. 
RICHARD Lionel (2012), Comprendre l'expressionnisme, Gollion, Infolio.

SARRAZAC Jean-Pierre (2012), Poétique du drame moderne, Paris, Seuil.

SARRAZAC Jean-Pierre (2018), Strindberg, L'Impersonnel, Paris, L'Arche.

STEFFENS Wilhelm (1993), "Thêâtre », (éd.) Lionel Richard, Encyclopédie de l'Expressionnisme, Paris, Somogy, p. 157-186.

STRINDBERG August (1964), (éd.) Maurice Gravier, Théâtre cruel et théâtre mystique, trad. Marguerite Diehl, Paris, Gallimard.

StRINDBERG August (1983), Le Chemin de Damas, trad. Alfred Jolivet, Maurice Gravier, Paris, L'Arche.

StRINDBERG August (2006), Le Songe, trad. André Markowicz, Frédéric Noguer, Beançon, Les Solitaires Intempestifs. 